\title{
Mean convergence theorems for weighted sums of random variables under a condition of weighted integrability
}

Lingqiu Cao*

*Correspondence: clq0621@sina.cn Common Required Course Department, Hengyang Financial and Industry Polytechnic, Hengyang, 421002, P.R. China

\begin{abstract}
In this paper, a new concept of weighted integrability is introduced for an array of random variables concerning an array of constants, which is weaker than other previous related notions of integrability. Mean convergence theorems for weighted sums of an array of dependent random variables satisfying this condition of integrability are obtained. Our results extend and sharpen the known results in the literature.

MSC: $60 \mathrm{~F} 15$

Keywords: mean convergence; weak laws of large numbers; uniform integrability; integrability concerning the weights; weighted sums; negatively quadrant dependent random variables; negatively associated random variables
\end{abstract}

\section{Introduction}

The notion of uniform integrability plays the central role in establishing weak laws of large numbers. In this paper, we introduce a new notion of weighted integrability and prove some weak laws of large numbers under this condition.

Definition 1.1 A sequence $\left\{X_{n}, n \geq 1\right\}$ of integrable random variables is said to be uniformly integrable if

$$
\lim _{a \rightarrow \infty} \sup _{n \geq 1} E\left|X_{n}\right| I\left(\left|X_{n}\right|>a\right)=0 .
$$

Landers and Rogge [1] proved the weak law of large numbers under the sequence of pairwise independent uniformly integrable random variables.

Chandra [2] obtained the weak law of large numbers under a new condition which is weaker than uniform integrability: Cesàro uniform integrability.

Definition 1.2 A sequence $\left\{X_{n}, n \geq 1\right\}$ of integrable random variables is said to be Cesàro uniformly integrable if

$$
\lim _{a \rightarrow \infty} \sup _{n \geq 1} \frac{1}{n} \sum_{i=1}^{n} E\left|X_{i}\right| I\left(\left|X_{i}\right|>a\right)=0 .
$$

๑ 2013 Cao; licensee Springer. This is an Open Access article distributed under the terms of the Creative Commons Attribution License (http://creativecommons.org/licenses/by/2.0), which permits unrestricted use, distribution, and reproduction in any medium, provided the original work is properly cited. 
In the following, let $\left\{u_{n}, n \geq 1\right\}$ and $\left\{v_{n}, n \geq 1\right\}$ be two sequences of integers (not necessary positive or finite) such that $v_{n}>u_{n}$ for all $n \geq 1$ and $v_{n}-u_{n} \rightarrow \infty$ as $n \rightarrow \infty$. Let $\left\{k_{n}, n \geq 1\right\}$ be a sequence of positive numbers such that $k_{n} \rightarrow \infty$ as $n \rightarrow \infty$.

Ordóñez Cabrera [3] introduced the concept of uniform integrability concerning an array of constant weights.

Definition 1.3 Let $\left\{X_{n i}, u_{n} \leq i \leq v_{n}, n \geq 1\right\}$ be an array of random variables and $\left\{a_{n i}, u_{n} \leq\right.$ $\left.i \leq v_{n}, n \geq 1\right\}$ be an array of constants with $\sum_{i=u_{n}}^{v_{n}}\left|a_{n i}\right| \leq C$ for all $n \in N$ and some constant $C>0$. The array $\left\{X_{n i}, u_{n} \leq i \leq v_{n}, n \geq 1\right\}$ is said to be $\left\{a_{n i}\right\}$-uniformly integrable if

$$
\lim _{a \rightarrow \infty} \sup _{n \geq 1} \sum_{i=u_{n}}^{v_{n}}\left|a_{n i}\right| E\left|X_{n i}\right| I\left(\left|X_{n i}\right|>a\right)=0 .
$$

Ordóñez Cabrera [3] proved that the condition of uniform integrability concerning an array of constant weights is weaker than uniform integrability, and leads to Cesàro uniform integrability as a special case. Under the condition of uniform integrability concerning the weights, he obtained the weak law of large numbers for weighted sums of pairwise independent random variables.

Sung [4] introduced the concept of Cesàro-type uniform integrability with exponent $r$.

Definition 1.4 Let $\left\{X_{n i}, u_{n} \leq i \leq v_{n}, n \geq 1\right\}$ be an array of random variables and $r>0$. The array $\left\{X_{n i}, u_{n} \leq i \leq v_{n}, n \geq 1\right\}$ is said to be Cesàro-type uniformly integrable with exponent $r$ if

$$
\sup _{n \geq 1} \frac{1}{k_{n}} \sum_{i=u_{n}}^{v_{n}} E\left|X_{n i}\right|^{r}<\infty \quad \text { and } \quad \lim _{a \rightarrow \infty} \sup _{n \geq 1} \frac{1}{k_{n}} \sum_{i=u_{n}}^{v_{n}} E\left|X_{n i}\right|^{r} I\left(\left|X_{n i}\right|^{r}>a\right)=0 \text {. }
$$

Note that the conditions of Cesàro uniform integrability and Cesàro-type uniform integrability with exponent $r$ are equivalent when $u_{n}=1, v_{n}=n, n \geq 1$ and $r=1$. Sung [4] obtained the weak law of large numbers for an array $\left\{X_{n i}\right\}$ satisfying Cesàro-type uniform integrability with exponent $r$ for some $0<r<2$.

Chandra and Goswami [5] introduced the concept of Cesàro $\alpha$-integrability $(\alpha>0)$ and showed that Cesàro $\alpha$-integrability, for any $\alpha>0$, is weaker than Cesàro uniform integrability.

Definition 1.5 Let $\alpha>0$. A sequence $\left\{X_{n}, n \geq 1\right\}$ of random variables is said to be Cesàro $\alpha$-integrable if

$$
\sup _{n \geq 1} \frac{1}{n} \sum_{i=1}^{n} E\left|X_{i}\right|<\infty \quad \text { and } \quad \lim _{n \rightarrow \infty} \frac{1}{n} \sum_{i=1}^{n} E\left|X_{i}\right| I\left(\left|X_{i}\right|>i^{\alpha}\right)=0 .
$$

Under the Cesàro $\alpha$-integrability condition for some $\alpha>\frac{1}{2}$, Chandra and Goswami [5] obtained the weak law of large numbers for a sequence of pairwise independent random variables. They also proved that Cesàro $\alpha$-integrability for appropriate $\alpha$ is also sufficient for the weak law of large numbers to hold for certain special dependent sequences of random variables. 
Ordóñez Cabrera and Volodin [6] introduced the notion of $h$-integrability for an array of random variables concerning an array of constant weights, and proved that this concept is weaker than Cesàro uniform integrability, $\left\{a_{n i}\right\}$-uniform integrability and Cesàro $\alpha$-integrability.

Definition 1.6 Let $\left\{X_{n i}, u_{n} \leq i \leq v_{n}, n \geq 1\right\}$ be an array of random variables and $\left\{a_{n i}, u_{n} \leq\right.$ $\left.i \leq v_{n}, n \geq 1\right\}$ be an array of constants with $\sum_{i=u_{n}}^{v_{n}}\left|a_{n i}\right| \leq C$ for all $n \in N$ and some constant $C>0$. Moreover, let $\{h(n), n \geq 1\}$ be an increasing sequence of positive constants with $h(n) \uparrow \infty$ as $n \uparrow \infty$. The array $\left\{X_{n i}, u_{n} \leq i \leq v_{n}, n \geq 1\right\}$ is said to be $h$-integrable with respect to the array of constants $\left\{a_{n i}\right\}$ if

$$
\sup _{n \geq 1} \sum_{i=u_{n}}^{v_{n}}\left|a_{n i}\right| E\left|X_{n i}\right|<\infty \quad \text { and } \quad \lim _{n \rightarrow \infty} \sum_{i=u_{n}}^{v_{n}}\left|a_{n i}\right| E\left|X_{n i}\right| I\left(\left|X_{n i}\right|>h(n)\right)=0
$$

Under appropriate conditions on the weights, Ordóñez Cabrera and Volodin [6] proved that $h$-integrability concerning the weights is sufficient for the weak law of large numbers to hold for weighted sums of an array of random variables, when these random variables are subject to some special kind of rowwise dependence.

Sung et al. [7] introduced the notion of $h$-integrability with exponent $r(r>0)$.

Definition 1.7 Let $\left\{X_{n i}, u_{n} \leq i \leq v_{n}, n \geq 1\right\}$ be an array of random variables and $r>0$. Moreover, let $\{h(n), n \geq 1\}$ be an increasing sequence of positive constants with $h(n) \uparrow \infty$ as $n \uparrow \infty$. The array $\left\{X_{n i}\right\}$ is said to be $h$-integrable with exponent $r$ if

$$
\sup _{n \geq 1} \frac{1}{k_{n}} \sum_{i=u_{n}}^{v_{n}} E\left|X_{n i}\right|^{r}<\infty \quad \text { and } \quad \lim _{n \rightarrow \infty} \frac{1}{k_{n}} \sum_{i=u_{n}}^{v_{n}} E\left|X_{n i}\right|^{r} I\left(\left|X_{n i}\right|^{r}>h(n)\right)=0 \text {. }
$$

Sung et al. [7] proved that $h$-integrability with exponent $r(r>0)$ is weaker than Cesàrotype uniform integrability with exponent $r$, and obtained weak law of large numbers for an array of dependent random variables (martingale difference sequence or negatively associated random variables ) satisfying the condition of $h$-integrability with exponent $r$.

Chandra and Goswami [8] introduced the concept of residual Cesàro $(\alpha, p)$ - integrability $(\alpha>0, p>0)$ and showed that residual Cesàro $(\alpha, p)$-integrability for any $\alpha>0$ is strictly weaker than Cesàro $\alpha$-integrability.

Definition 1.8 Let $\alpha>0, p>0$. A sequence $\left\{X_{n}, n \geq 1\right\}$ of random variables is said to be residually Cesàro $(\alpha, p)$-integrable if

$$
\sup _{n \geq 1} \frac{1}{n} \sum_{i=1}^{n} E\left|X_{i}\right|^{p}<\infty \quad \text { and } \quad \lim _{n \rightarrow \infty} \frac{1}{n} \sum_{i=1}^{n} E\left(\left|X_{i}\right|-i^{\alpha}\right)^{p} I\left(\left|X_{i}\right|>i^{\alpha}\right)=0 .
$$

Under the residual Cesàro $(\alpha, p)$-integrability condition for some appropriate $\alpha$ and $p$, Chandra and Goswami [8] obtained $L^{1}$-convergence and the weak law of large numbers for a sequence of dependent random variables.

We now introduce a new concept of integrability. 
Definition 1.9 Let $r>0$ and $\left\{X_{n i}, u_{n} \leq i \leq v_{n}, n \geq 1\right\}$ be an array of random variables. Moreover, let $\left\{a_{n i}, u_{n} \leq i \leq v_{n}, n \geq 1\right\}$ be an array of constants and $\{h(n), n \geq 1\}$ an increasing sequence of positive constants with $h(n) \uparrow \infty$ as $n \uparrow \infty$. The array $\left\{X_{n i}, u_{n} \leq i \leq v_{n}, n \geq\right.$ $1\}$ is said to be residually $(r, h)$-integrable with respect to the array of constants $\left\{a_{n i}\right\}$ if

$$
\sup _{n \geq 1} \sum_{i=u_{n}}^{v_{n}}\left|a_{n i}\right|^{r} E\left|X_{n i}\right|^{r}<\infty \quad \text { and } \quad \lim _{n \rightarrow \infty} \sum_{i=u_{n}}^{v_{n}}\left|a_{n i}\right|^{r} E\left(\left|X_{n i}\right|-h(n)\right)^{r} I\left(\left|X_{n i}\right|>h(n)\right)=0 .
$$

Remark 1.1 (i) The residual $(1, h)$-integrability concerning the arrays of constants was defined by Yuan and Tao [9], who called it the residual $h$-integrability, and was extended by Ordóñez Cabrera et al. [10] to the conditionally residually $h$-integrability relative to a sequence of $\sigma$-algebras.

(ii) If $\left\{X_{n i}, u_{n} \leq i \leq v_{n}, n \geq 1\right\}$ is $h$-integrable with exponent $r$, then it is residually $\left(r, h^{1 / r}\right)$ integrable with respect to the array of constants $\left\{a_{n i}\right\}$ satisfying $a_{n i}=k_{n}^{-1 / r}, u_{n} \leq i \leq v_{n}$, $n \geq 1$.

(iii) Residually $(r, h)$-integrable with respect to the array of constants $\left\{a_{n i}\right\}$ is weaker than residually Cesàro $(\alpha, p)$-integrable.

(iv) The concept of residually $(r, h)$-integrable concerning the array of constants $\left\{a_{n i}\right\}$ is strictly weaker than the concept of $h$-integrable concerning the array of constants $\left\{a_{n i}\right\}$ and $h$-integrable with exponent $r$.

Therefore, the concept of residually $(r, h)$-integrable concerning the array of constants $\left\{a_{n i}\right\}$ is weaker than the concept of all Definitions 1.1-1.7, and leads to residual Cesàro $(\alpha, p)$-integrability as a special case.

For the array $\left\{X_{n i}, u_{n} \leq i \leq v_{n}, n \geq 1\right\}$ of random variables, weak laws of large numbers have been established by many authors (referring to: Sung et al. [7, 11]; Sung [4]; Ordóñez Cabrera and Volodin [6]).

In this paper, we obtain weak laws of large numbers for the array of dependent random variables satisfying the condition of residually $(r, h)$-integrable with respect to the array of constants $\left\{a_{n i}\right\}$. Our results extend and sharpen the results of Sung et al. [7], Sung et al. [11], Sung [4], Ordóñez Cabrera and Volodin [6].

\section{Preliminary lemmas}

In order to consider the mean convergence for an array of random variables satisfying dependent conditions, we need the following definition.

Definition 2.1 Two random variables $X$ and $Y$ are said to be negatively quadrant dependent (NQD) or lower case negatively dependent (LCND) if

$$
P(X \leq x, Y \leq y) \leq P(X \leq x) P(Y \leq y) \quad \forall x, y \in R .
$$

An infinite family of random variables $\left\{X_{n}, n \geq 1\right\}$ is said to be pairwise NQD if every two random variables $X_{i}$ and $X_{j}(i \neq j)$ are NQD. The array $\left\{X_{n i}, i \geq 1, n \geq 1\right\}$ is said to be rowwise pairwise NQD if every positive integer $n$, the sequence of random variables $\left\{X_{n i}, i \geq 1\right\}$ is pairwise NQD.

This definition was introduced by Alam and Saxena [12] and carefully studied by JoagDev and Proschan [13]. 
Lemma 2.1 Let $\left\{X_{n}, n \geq 1\right\}$ be a sequence of pairwise $N Q D$ random variables. Let $\left\{f_{n}, n \geq 1\right\}$ be a sequence of increasing functions. Then $\left\{f_{n}\left(X_{n}\right), n \geq 1\right\}$ is a sequence of pairwise NQD random variables.

If random variables $X$ and $Y$ are NQD, then $E(X Y) \leq E X E Y$, so we have the following.

Lemma 2.2 Let $\left\{X_{n}, n \geq 1\right\}$ be a sequence of pairwise $N Q D$ random variables with $E X_{n}=0$ and $E X_{n}^{2}<\infty, n \geq 1$. Then

$$
E\left(\sum_{i=1}^{n} X_{i}\right)^{2} \leq \sum_{i=1}^{n} E X_{i}^{2}
$$

Using the above lemma, Chen et al. [14] obtained the following inequality.

Lemma 2.3 Let $\left\{X_{n}, n \geq 1\right\}$ be a sequence of pairwise $N Q D$ random variables with $E X_{n}=0$ and $E\left|X_{n}\right|^{p}<\infty, n \geq 1$, where $1 \leq p \leq 2$. Then

$$
E\left|\sum_{i=1}^{n} X_{i}\right|^{p} \leq c_{p} \sum_{i=1}^{n} E\left|X_{i}\right|^{p}, \quad \forall n \geq 2,
$$

where $c_{p}>0$ depends only on $p$.

\section{Main results and proofs}

Theorem 3.1 Let $0<r<2$ and $\left\{X_{n i}, u_{n} \leq i \leq v_{n}, n \geq 1\right\}$ be an array of random variables. Let $\left\{a_{n i}, u_{n} \leq i \leq v_{n}, n \geq 1\right\}$ be an array of constants and $\{h(n), n \geq 1\}$ an increasing sequence of positive constants with $h(n) \uparrow \infty$ as $n \uparrow \infty$. Assume that the following conditions hold:

(i) $\left\{X_{n i}, u_{n} \leq i \leq v_{n}, n \geq 1\right\}$ is residually $(r, h)$-integrable concerning the array $\left\{a_{n i}\right\}$;

(ii) $h(n) \sup _{u_{n} \leq i \leq v_{n}}\left|a_{n i}\right| \rightarrow 0$.

Then

$$
\sum_{i=u_{n}}^{v_{n}} a_{n i}\left(X_{n i}-b_{n i}\right) \rightarrow 0
$$

in $L^{r}$ and, hence, in probability as $n \rightarrow \infty$, where $b_{n i}=0$ if $0<r<1$ and $b_{n i}=E\left(X_{n i} \mid \Im_{n, i-1}\right)$ if $1 \leq r<2$, where $\Im_{n, i}=\sigma\left(X_{n i}, u_{n} \leq j \leq i\right), u_{n} \leq i \leq v_{n}, n \geq 1$, and $\Im_{n, u_{n}-1}=\{\emptyset, \Omega\}, n \geq 1$.

Proof If $u_{n}=-\infty$ and/or $v_{n}=+\infty$, by the $C_{r}$-inequality, Jensen's inequality and $0<r<2$, we have

$$
\sup _{n \geq 1} \sum_{i=u_{n}}^{v_{n}}\left|a_{n i}\right|^{r} E\left|X_{n i}-b_{n i}\right|^{r} \leq 2 \sup _{n \geq 1} \sum_{i=u_{n}}^{v_{n}}\left|a_{n i}\right|^{r} E\left|X_{n i}\right|^{r}<\infty .
$$

Therefore, if $0<r<1$, we have $E\left(\sum_{i=u_{n}}^{v_{n}}\left|a_{n i} X_{n i}\right|\right)^{r} \leq \sum_{i=u_{n}}^{v_{n}}\left|a_{n i}\right|^{r} E\left|X_{n i}\right|^{r}<\infty$, so $\sum_{i=u_{n}}^{v_{n}} a_{n i} X_{n i}$ a.s. converges for all $n \geq 1$. If $1 \leq r<2$, by Theorem 2.17 of Hall and Heyde [15], we can get that $\sum_{i=u_{n}}^{v_{n}} a_{n i}\left(X_{n i}-b_{n i}\right)$ a.s. converges for all $n \geq 1$. Thus $\sum_{i=u_{n}}^{v_{n}} a_{n i}\left(X_{n i}-b_{n i}\right)$ a.s. converges for all $n \geq 1$ in the case of $u_{n}=-\infty$ and/or $v_{n}=+\infty$. 
Let $X_{n i}^{\prime}=X_{n i} I\left(\left|X_{n i}\right| \leq h(n)\right)-h(n) I\left(X_{n i}<-h(n)\right)+h(n) I\left(X_{n i}>h(n)\right)$ and $X_{n i}^{\prime \prime}=X_{n i}-X_{n i}^{\prime}=$ $\left(X_{n i}+h(n)\right) I\left(X_{n i}<-h(n)\right)+\left(X_{n i}-h(n)\right) I\left(X_{n i}>h(n)\right)$ for $u_{n} \leq i \leq u_{n}, n \geq 1$.

Case $0<r<1$. By the $C_{r}$-inequality, we obtain

$$
E\left|\sum_{i=u_{n}}^{v_{n}} a_{n i} X_{n i}\right|^{r} \leq E\left|\sum_{i=u_{n}}^{v_{n}} a_{n i} X_{n i}^{\prime}\right|^{r}+E\left|\sum_{i=u_{n}}^{v_{n}} a_{n i} X_{n i}^{\prime \prime}\right|^{r}
$$

Noting that $\left|X_{n i}^{\prime \prime}\right| \leq\left(\left|X_{n i}\right|-h(n)\right) I\left(\left|X_{n i}\right|>h(n)\right)$ for all $u_{n} \leq i \leq v_{n}, n \geq 1$, by the $C_{r^{-}}$ inequality, we obtain

$$
\begin{aligned}
E\left|\sum_{i=u_{n}}^{v_{n}} a_{n i} X_{n i}^{\prime \prime}\right|^{r} & \leq \sum_{i=u_{n}}^{v_{n}}\left|a_{n i}\right|^{r} E\left|X_{n i}^{\prime \prime}\right|^{r} \\
& \leq \sum_{i=u_{n}}^{v_{n}}\left|a_{n i}\right|^{r} E\left(\left|X_{n i}\right|-h(n)\right)^{r} I\left(\left|X_{n i}\right|>h(n)\right) \rightarrow 0 .
\end{aligned}
$$

Since $\left|X_{n i}^{\prime}\right| \leq \min \left\{\left|X_{n i}\right|, h(n)\right\}$ and $0<r<1$, we have

$$
\begin{aligned}
E\left|\sum_{i=u_{n}}^{v_{n}} a_{n i} X_{n i}^{\prime}\right| & \leq \sum_{i=u_{n}}^{v_{n}}\left|a_{n i}\right| E\left|X_{n i}^{\prime}\right| \\
& \leq C\left(h(n) \sup _{u_{n} \leq i \leq v_{n}}\left|a_{n i}\right|\right)^{1-r} \sum_{i=u_{n}}^{v_{n}}\left|a_{n i}\right|^{r} E\left|X_{n i}\right|^{r} \rightarrow 0 .
\end{aligned}
$$

So, $\sum_{i=u_{n}}^{v_{n}} a_{n i} X_{n i}^{\prime} \rightarrow 0$ in $L^{1}$ and hence in $L^{r}$. Therefore, the proof is completed when $0<$ $r<1$.

Case $r=1$. Since

$$
\begin{aligned}
& E\left|\sum_{i=u_{n}}^{v_{n}} a_{n i}\left[X_{n i}-E\left(X_{n i} \mid \mathfrak{\Im}_{n, i-1}\right)\right]\right| \\
& \quad \leq E\left|\sum_{i=u_{n}}^{v_{n}} a_{n i}\left[X_{n i}^{\prime}-E\left(X_{n i}^{\prime} \mid \Im_{n, i-1}\right)\right]\right|+E\left|\sum_{i=u_{n}}^{v_{n}} a_{n i}\left[X_{n i}^{\prime \prime}-E\left(X_{n i}^{\prime \prime} \mid \mathfrak{\Im}_{n, i-1}\right)\right]\right| \\
& \quad \leq E\left|\sum_{i=u_{n}}^{v_{n}} a_{n i}\left[X_{n i}^{\prime}-E\left(X_{n i}^{\prime} \mid \Im_{n, i-1}\right)\right]\right|+2 E \sum_{i=u_{n}}^{v_{n}}\left|a_{n i} X_{n i}^{\prime \prime}\right| .
\end{aligned}
$$

But

$$
E \sum_{i=u_{n}}^{v_{n}}\left|a_{n i} X_{n i}^{\prime \prime}\right| \leq \sum_{i=u_{n}}^{v_{n}}\left|a_{n i}\right| E\left(\left|X_{n i}\right|-h(n)\right) I\left(\left|X_{n i}\right|>h(n)\right) \rightarrow 0
$$

By Burkholder's inequality (Theorem 2.10 of Hall and Heyde [15]), we have

$$
\begin{aligned}
& E\left|\sum_{i=u_{n}}^{v_{n}} a_{n i}\left[X_{n i}^{\prime}-E\left(X_{n i}^{\prime} \mid \Im_{n, i-1}\right)\right]\right|^{2} \\
& \quad \leq C \sum_{i=u_{n}}^{v_{n}} a_{n i}^{2} E\left[X_{n i}^{\prime}-E\left(X_{n i}^{\prime} \mid \Im_{n, i-1}\right)\right]^{2}
\end{aligned}
$$




$$
\begin{aligned}
& \leq C E \sum_{i=u_{n}}^{v_{n}} a_{n i}^{2} E\left|X_{n i}^{\prime}\right|^{2} \\
& \leq C\left(h(n) \sup _{u_{n} \leq i \leq v_{n}}\left|a_{n i}\right|\right) \sum_{i=u_{n}}^{v_{n}}\left|a_{n i}\right| E\left|X_{n i}\right| \rightarrow 0,
\end{aligned}
$$

hereinafter, $C$ always stands for a positive constant not depending on $n$ which may differ from one place to another, thus $\sum_{i=u_{n}}^{v_{n}} a_{n i}\left[X_{n i}^{\prime}-E\left(X_{n i}^{\prime} \mid \Im_{n, i-1}\right)\right] \rightarrow 0$ in $L^{2}$ and hence in $L^{1}$. Therefore, the proof is completed when $r=1$.

Case $1<r<2$. By Burkholder's inequality, the $C_{r}$-inequality and Jensen's inequality, we have

$$
\begin{gathered}
E\left|\sum_{i=u_{n}}^{v_{n}} a_{n i}\left[X_{n i}-E\left(X_{n i} \mid \Im_{n, i-1}\right)\right]\right|^{r} \\
\leq C E\left|\sum_{i=u_{n}}^{v_{n}} a_{n i}^{2}\left[X_{n i}-E\left(X_{n i} \mid \Im_{n, i-1}\right)\right]^{2}\right|^{r / 2} \\
\leq C E\left|\sum_{i=u_{n}}^{v_{n}} a_{n i}^{2}\left[X_{n i}^{\prime}-E\left(X_{n i}^{\prime} \mid \Im_{n, i-1}\right)\right]^{2}\right|^{r / 2} \\
+C E \sum_{i=u_{n}}^{v_{n}}\left|a_{n i}\right|^{r}\left|\left[X_{n i}^{\prime \prime}-E\left(X_{n i}^{\prime \prime} \mid \Im_{n, i-1}\right)\right]\right|^{r} \\
\leq C\left\{\sum_{i=u_{n}}^{v_{n}} a_{n i}^{2} E\left|X_{n i}^{\prime}\right|^{2}\right\}^{r / 2}+C \sum_{i=u_{n}}^{v_{n}} E\left|a_{n i} X_{n i}^{\prime \prime}\right|^{r} .
\end{gathered}
$$

But

$$
\sum_{i=u_{n}}^{v_{n}} a_{n i}^{2} E\left|X_{n i}^{\prime}\right|^{2} \leq\left(h(n) \sup _{u_{n} \leq i \leq v_{n}}\left|a_{n i}\right|\right)^{2-r} \sum_{i=u_{n}}^{v_{n}}\left|a_{n i}\right|^{r} E\left|X_{n i}\right|^{r} \rightarrow 0,
$$

and

$$
\sum_{i=u_{n}}^{v_{n}} E\left|a_{n i} X_{n i}^{\prime \prime}\right|^{r} \leq \sum_{i=u_{n}}^{v_{n}}\left|a_{n i}\right|^{r} E\left(\left|X_{n i}\right|-h(n)\right)^{r} I\left(\left|X_{n i}\right|>h(n)\right) \rightarrow 0 .
$$

Therefore, the proof is completed when $1<r<2$.

Remark 3.1 (i) Putting $a_{n i}=k_{n}^{-1 / r}, u_{n} \leq i \leq v_{n}, n \geq 1$, if $\left\{X_{n i}, u_{n} \leq i \leq v_{n}, n \geq 1\right\}$ is an array of $h$-integrability with exponent $r(0<r<2)$, then it is residually $\left(r, h^{1 / r}\right)$-integrable concerning the array $\left\{a_{n i}\right\}$. Thus, Theorem 3.1 and Corollary 3.1 of Sung and Lisawadi and Volodin [7] can be obtained from Theorem 3.1.

(ii) Let $a_{n i}=1 / n^{r}, u_{n}=1, v_{n}=n, n \geq 1, h(n)=n^{\alpha}, \alpha \in(0,1 / r)$, similar to that of Remark 1 of Ordóñez Cabrera and Volodin [6], Theorem 3.1 and 3.2 and Corollary 3.1 of Chandra and Goswami [8] can be obtained from Theorem 3.1.

Theorem 3.2 Let $1 \leq r<2$ and $\left\{X_{n i}, u_{n} \leq i \leq v_{n}, n \geq 1\right\}$ be an array of rowwise pairwise $N Q D$ random variables. Let $\left\{a_{n i}, u_{n} \leq i \leq v_{n}, n \geq 1\right\}$ be an array of constants and $\{h(n), n \geq$ 
1) an increasing sequence of positive constants with $h(n) \uparrow \infty$ as $n \uparrow \infty$. Assume that the following conditions hold:

(i) $\left\{X_{n i}, u_{n} \leq i \leq v_{n}, n \geq 1\right\}$ is residually $(r, h)$-integrable concerning the array $\left\{a_{n i}\right\}$.

(ii) $h(n) \sup _{u_{n} \leq i \leq v_{n}}\left|a_{n i}\right| \rightarrow 0$.

Then

$$
\sum_{i=u_{n}}^{v_{n}} a_{n i}\left(X_{n i}-E X_{n i}\right) \rightarrow 0
$$

in $L^{r}$ and hence in probability as $n \rightarrow \infty$.

Proof The proof is similar to that of Theorem 3.1, we can get $\sum_{i=u_{n}}^{v_{n}} a_{n i}\left(X_{n i}-E X_{n i}\right)$ a.s. converges for all $n \geq 1$ in the case of $u_{n}=-\infty$ and/or $v_{n}=+\infty$. Let $X_{n i}^{\prime}$ and $X_{n i}^{\prime \prime}$ as in Theorem 3.1. Without loss of generality, we can assume that $a_{n i}>0$ for $u_{n} \leq i \leq v_{n}, n \geq 1$, then $\left\{a_{n i} X_{n i}^{\prime}, u_{n} \leq i \leq v_{n}, n \geq 1\right\}$ and $\left\{a_{n i} X_{n i}^{\prime \prime}, u_{n} \leq i \leq v_{n}, n \geq 1\right\}$ are arrays of rowwise NQD random variables by Lemma 2.1. Observe that

$$
\begin{aligned}
\sum_{i=u_{n}}^{v_{n}} a_{n i}\left(X_{n i}-E X_{n i}\right) & =\sum_{i=u_{n}}^{v_{n}} a_{n i}\left(X_{n i}^{\prime}-E X_{n i}^{\prime}\right)+\sum_{i=u_{n}}^{v_{n}} a_{n i}\left(X_{n i}^{\prime \prime}-E X_{n i}^{\prime \prime}\right) \\
& =: A_{n}+B_{n} .
\end{aligned}
$$

By Lemma 2.3 and $\left|X_{n i}^{\prime}\right| \leq \min \left\{\left|X_{n i}\right|, h(n)\right\}$, we have

$$
\begin{aligned}
E\left(A_{n}\right)^{2} & \leq C \sum_{i=u_{n}}^{v_{n}} a_{n i}^{2} E\left(X_{n i}^{\prime}-E X_{n i}^{\prime}\right)^{2} \\
& \leq C \sum_{i=u_{n}}^{v_{n}} a_{n i}^{2} E\left(X_{n i}^{\prime}\right)^{2} \\
& \leq C\left(h(n) \sup _{u_{n} \leq i \leq v_{n}}\left|a_{n i}\right|\right)^{2-r} \sum_{i=u_{n}}^{v_{n}}\left|a_{n i}\right|^{r} E\left|X_{n i}\right|^{r} \rightarrow 0,
\end{aligned}
$$

then $A_{n} \rightarrow 0$ in $L^{2}$ and hence in $L^{r}$.

By Lemma 2.3 and $\left|X_{n i}^{\prime \prime}\right| \leq\left(\left|X_{n i}\right|-h(n)\right) I\left(\left|X_{n i}\right|>h(n)\right)$, we have

$$
\begin{aligned}
E\left|B_{n}\right|^{r} & \leq C \sum_{i=u_{n}}^{v_{n}}\left|a_{n i}\right|^{r} E\left(X_{n i}^{\prime \prime}-E X_{n i}^{\prime \prime}\right)^{r} \\
& \leq C \sum_{i=u_{n}}^{v_{n}}\left|a_{n i}\right|^{r} E\left|X_{n i}^{\prime \prime}\right|^{r} \\
& \leq C \sum_{i=u_{n}}^{v_{n}}\left|a_{n i}\right|^{r} E\left(\left|X_{n i}\right|-h(n)\right)^{r} I\left(\left|X_{n i}\right|>h(n)\right) \rightarrow 0 .
\end{aligned}
$$

Thus, the proof is completed.

Remark 3.2 Theorem 3.2 extended the result in Chen [16] who first obtained the $r$-the moment convergence under the $r$ th uniform integrability for pairwise NQD sequence. 


\section{Remark 3.3}

(i) Let $r=1, a_{n i}=k_{n}^{-1}, u_{n} \leq i \leq v_{n}, n \geq 1$, then Theorem 3.2, Corollary 3.2 of Sung et al. [7] and Theorem 2.2 of Yuan and Tao [9] can be obtained from Theorem 3.2.

(ii) Theorem 2.2 of Chandra and Goswami [8] can be obtained from Theorem 3.2.

(iii) Theorem 1 and Corollary 1 of Ordóñez Cabrera and Volodin [6] can be obtained from Theorem 3.2.

Remark 3.4 Putting $a_{n i}=k_{n}^{-1 / r}, u_{n} \leq i \leq v_{n}, n \geq 1$, if $\left\{X_{n i}, u_{n} \leq i \leq v_{n}, n \geq 1\right\}$ is an array of $h$-integrability with exponent $r(0<r<2)$, then $\left\{X_{n i}, u_{n} \leq i \leq v_{n}, n \geq 1\right\}$ is residually $\left(r, h^{1 / r}\right)$-integrable concerning the array $\left\{a_{n i}\right\}$. Theorem 3.3 and Corollary 3.3 of Sung et al. [7] can be obtained from Theorem 3.2 since an NA sequence is an NQD sequence.

\section{Competing interests}

The author declares that they have no competing interests.

\section{Acknowledgements}

The authors are very grateful to the referees and the editors for their valuable comments and some helpful suggestions that improved the clarity and readability of the paper.

Received: 4 May 2013 Accepted: 21 October 2013 Published: 25 Nov 2013

\section{References}

1. Landers, $D$, Rogge, L: Laws of large numbers for pairwise independent uniformly integrable random variables. Math. Nachr. 130, 189-192 (1987)

2. Chandra, TK: Uniform integrability in the Cesàro sense and the weak law of large numbers. Sankhya, Ser. A 51, 309-317 (1989)

3. Ordóñez Cabrera, M: Convergence of weighted sums of random variables and uniform integrability concerning the weights. Collect. Math. 45(2), 121-132 (1994)

4. Sung, SH: Weak law of large numbers for arrays of random variables. Stat. Probab. Lett. 42(3), 293-298 (1999)

5. Chandra, TK, Goswami, A: Cesàro $\alpha$-integrability and laws of large numbers I. J. Theor. Probab. 16(3), 655-669 (2003)

6. Ordóñez Cabrera, M, Volodin, A: Mean convergence theorems and weak laws of large numbers for weighted sums of random variables under a condition of weighted integrability. J. Math. Anal. Appl. 305(2), 644-658 (2005)

7. Sung, SH, Lisawadi, S, Volodin, A: Weak laws of large numbers for arrays under a condition of uniform integrability. J. Korean Math. Soc. 45(1), 289-300 (2008)

8. Chandra, TK, Goswami, A: Cesàro $\alpha$-integrability and laws of large numbers-II. J. Theor. Probab. 19(4), 789-816 (2006)

9. Yuan, $D$, Tao, B: Mean convergence theorems for weighted sums of arrays of residually $h$-integrable random variables concerning the weights under dependent assumptions. Acta Appl. Math. 103, 221-234 (2008)

10. Ordóñez Cabrera, M, Rosalsky, A, Volodin, A: Some theorems on conditional mean convergence and conditional almost sure convergence for randomly weighted sums of dependent random variables. Test 21, 369-385 (2012)

11. Sung, SH, Hu, TC, Volodin, A: On the weak laws for arrays of random variables. Stat. Probab. Lett. 72(4), 291-298 (2005)

12. Alam, K, Saxena, KML: Positive dependence in multivariate distributions. Commun. Stat., Theory Methods 10 , 1183-1196 (1981)

13. Joag-Dev, K, Proschan, F: Negative association of random variables with applications. Ann. Stat. 11, 286-295 (1983)

14. Chen, P, Bai, P, Sung, SH: The von Bahr-Esseen moment inequality for pairwise independent random variables and applications (to appear)

15. Hall, P, Heyde, CC: Martingale Limit Theory and Its Application. Academic Press, New York (1980)

16. Chen, P: $L^{\prime}$ convergence for pairwise NQD random variables. Acta Math. Sci. 28, 447-453 (2008) (in Chinese)

10.1186/1029-242X-2013-558

Cite this article as: Cao: Mean convergence theorems for weighted sums of random variables under a condition of weighted integrability. Journal of Inequalities and Applications 2013, 2013:558 